\title{
Inhibition of miR-1193 leads to synthetic lethality in glioblastoma multiforme cells deficient of DNA-PKCS
}

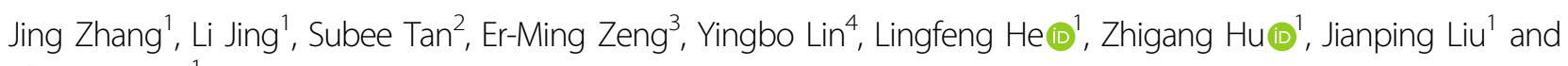
Zhigang Guo'

\begin{abstract}
Glioblastoma multiforme (GBM) is the most malignant primary brain tumor and has the highest mortality rate among cancers and high resistance to radiation and cytotoxic chemotherapy. Although some targeted therapies can partially inhibit oncogenic mutation-driven proliferation of GBM cells, therapies harnessing synthetic lethality are 'coincidental' treatments with high effectiveness in cancers with gene mutations, such as GBM, which frequently exhibits DNA-PKcs mutation. By implementing a highly efficient high-throughput screening (HTS) platform using an in-house-constructed genome-wide human microRNA inhibitor library, we demonstrated that miR-1193 inhibition sensitized GBM tumor cells with DNA-PKcs deficiency. Furthermore, we found that miR-1193 directly targets YY1AP1, leading to subsequent inhibition of FEN1, an important factor in DNA damage repair. Inhibition of miR-1193 resulted in accumulation of DNA double-strand breaks and thus increased genomic instability. RPA-coated SsDNA structures enhanced ATR checkpoint kinase activity, subsequently activating the CHK1/p53/apoptosis axis. These data provide a preclinical theory for the application of miR-1193 inhibition as a potential synthetic lethal approach targeting GBM cancer cells with DNA-PKcs deficiency.
\end{abstract}

\section{Introduction}

Glioblastoma multiforme (GBM), exhibits highly aggressive invasion, a high mortality rate, and high resistance to radiation and cytotoxic chemotherapy, and thus is the most common malignant primary brain tumor and is not surgically curable ${ }^{1}$. Despite the introduction of modern therapeutic approaches, the median overall survival time of GBM patients is less than 1 year $^{2}$. Similar to normal cells, tumor cells are subject to DNA damage, particularly damage from double-strand breaks (DSBs) ${ }^{3}$.

\footnotetext{
Correspondence: Jing Zhang (jingzhang1006@gmail.com) or

Jianping Liu (liujianping88@hotmail.com) or

Zhigang Guo (guozgang@gmail.com)

'Jiangsu Key Laboratory for Molecular and Medical Biotechnology, College of Life Sciences, Nanjing Normal University, 210097 Nanjing, Jiangsu, P.R. China ${ }^{2}$ Key Laboratory for Molecular Biotechnology, College of Life Sciences, Nanjing University, 210093 Nanjing, Jiangsu, P.R. China

Full list of author information is available at the end of the article

Edited by M. Agostini
}

In response to DNA damage, cells activate the DNA damage response (DDR) network, allowing DNA repair through the regulation of cell-cycle progression, DNA damage repair or apoptosis ${ }^{4}$. DNA-dependent protein kinase catalytic subunit (DNA-PKcs) is required for DSB repair via the nonhomologous end joining (NHEJ) pathway, while DNA-PKcs deficiency constitutes one of the most typical features of $\mathrm{GBMs}^{5}$. However, the factors that can enhance the survival of DNA-PKcs-deficient GBM cells remain unknown. An alternative DSB repair mechanism, such as homologous recombination (HR) or microhomology-mediated end joining (MMEJ), which is also involved in DSB repair, may compensate for NHEJ pathway deficiency ${ }^{6,7}$. These DSB repair pathways comprise multiple components, such as ataxia-telangiectasia mutated (ATM), ATR, RAD51, BRAC1, and FEN1 ${ }^{3,8,9}$. $\mathrm{HR}$ requires the function of ATM, but the level of ATM is low in GBM cells due to the high expression of miR-

\section{(c) The Author(s) 2020}

(c) (i) Open Access This article is licensed under a Creative Commons Attribution 4.0 International License, which permits use, sharing, adaptation, distribution and reproduction cc) in any medium or format, as long as you give appropriate credit to the original author(s) and the source, provide a link to the Creative Commons license, and indicate if changes were made. The images or other third party material in this article are included in the article's Creative Commons license, unless indicated otherwise in a credit line to the material. If material is not included in the article's Creative Commons license and your intended use is not permitted by statutory regulation or exceeds the permitted use, you will need to obtain permission directly from the copyright holder. To view a copy of this license, visit http://creativecommons.org/licenses/by/4.0/. 
$100^{10,11}$. The term synthetic lethality describes a phenomenon in which simultaneous perturbation of two (or more) functionally complementary genes results in loss of cell viability ${ }^{12-14}$. The application of PARP inhibitors as components in synthetic lethal treatments pioneered a promising clinical therapeutic strategy for breast cancers with mutations in BRCA1 or BRCA $2^{15}$. Moreover, other synthetic lethal interactions have suggested more potential specific anticancer drug targets ${ }^{16-19}$.

MicroRNAs (miRNAs or miRs) are small, highly conserved, noncoding RNA molecules that usually repress gene translation by binding to complementary sequences in the $3^{\prime}$ untranslated regions ( $\left.3^{\prime} \mathrm{UTRs}\right)$ of their target mRNAs ${ }^{20}$. Accumulating evidence indicates that miRNAs play crucial roles in the establishment, progression and recurrence of human cancers ${ }^{21-24}$. In addition, many studies have demonstrated synthetic lethal interactions between miRNAs and specific genes in various cancer cells. For instance, synthetic lethality between the miR-1792 cluster and the tumor suppressor p53 was documented to suppress retinoblastoma formation; ${ }^{25}$ synthetic lethality between miR-206 and c-Myc via direct inhibition of MAP3K13 was also identified ${ }^{26}$. To date, targeting miRNAs may represent an appealing approach for synthetic lethal targeting of cancer cells with a specific genetic deficiency.

As a structure-specific endonuclease, Flap endonuclease 1 (FEN1) plays vital roles in DSB repair via HR and MMEJ, as well as through the long patch base excision repair (LP-BER) pathway ${ }^{19,27,28}$. A higher level of FEN1 expression was detected in rapidly dividing cells, including various types of cancer cells, than in cells with normal division kinetics and is associated with enhanced malignancy and decreased survival rates ${ }^{29-31}$. In addition, robust evidence revealed that silencing FEN1 leads to enhanced cisplatin sensitivity in glioma ${ }^{32}$, indicating a potential strategy for glioma cancer treatment.

In this study, to identify miRNAs that exhibit synthetic lethality with DNA-PKcs, we implemented an efficient genome-wide high-throughput screening (HTS) method to screen an in-house-constructed antisense oligonucleotide library against 2590 human miRNAs. The miR1193/FEN1 signaling pathway was identified as a promising synthetic lethal regulator in DNA-PKcs-deficient GBM cells. Moreover, disruption of the DNA-PKcs/miR1193/FEN1 axis may exert anticancer effects.

\section{Materials and methods}

\section{Cell lines and transfection}

U118MG, M059J, M059K, U251, A549, HepG2, Huh7, RPE-1, $293 \mathrm{~T}$ cells, and fibroblasts were purchased from the American Type Culture Collection. Cells free of mycoplasma contamination were maintained in Dulbec- co's modified Eagle's medium (DMEM) or a 1:1 mixture of DMEM and Ham's F12 medium supplemented with $2.5 \mathrm{mM}$ L-glutamine, $15 \mathrm{mM}$ HEPES, $0.5 \mathrm{mM}$ sodium pyruvate, $1.2 \mathrm{~g} / \mathrm{L}$ sodium bicarbonate, $0.05 \mathrm{mM}$ nonessential amino acids and 10\% fetal bovine serum. Cells were cultured at $37^{\circ} \mathrm{C}$ in a humidified atmosphere containing $5 \% \mathrm{CO}_{2}$. Cells were seeded in 6-well plates and transfected with anti-miR-1193 (antagomir, antisense oligonucleotide with the reverse complementary sequence of miR-1193), anti-miR-NC (neutral control), shFEN1, siDNA-PKcs, ORF DNA-PKcs, siYY1AP1, and ORF YY1AP1 (purchased from Ruibo, Guangzhou China) by using Lipofectamine 2000 (Invitrogen, USA) according to the manufacturer's instructions. Cells were harvested on day 4 after transfection for further analyses.

\section{High-throughput screening (HTS)}

Methods similar to those previously described were used $^{33}$. In brief, reverse complimentary sequences of 2590 individual mature human miRNAs were synthesized as a genome-wide miRNA inhibitor library (purchased from Ruibo, Guangzhou, China). U118MG and M059J cells (800 cells per well in $50 \mu \mathrm{l}$ of medium) were transfected separately with each of the 2590 miRNA antisense oligonucleotides (final concentration, $100 \mu \mathrm{M}$ ) in black polystyrene 384-well flat-bottom microtiter plates (Becton Dickinson, NJ, USA) using a Multidrop ${ }^{\mathrm{TM}}$ Combi Reagent Dispenser (Thermo Fisher Scientific, MA, USA). On day 4 , cells were fixed with $4 \%$ paraformaldehyde solution and stained with $1 \mathrm{mg} / \mathrm{ml}$ Hoechst (Sigma-Aldrich, MA, USA) for $15 \mathrm{~min}$ at room temperature. Each well was subjected to automated image acquisition for nuclei counting at acumen ${ }^{\circledR}$ Cellista laser scanning imaging cytometer (TTP Labtech, UK).

\section{Hit selection}

Antisense oligonucleotides inhibiting miRNAs were selected as hits based on the nuclei count in the U118MG and M059J cell lines. The standard deviation was obtained for each 384-well plate from the median absolute deviation of the number of nuclei in all treated wells. To calculate the Z score of each miRNA inhibitor, we used the population mean and standard deviation for calculation as $\mathrm{z}=(\mathrm{x}-\mu) / \sigma$, where $\mathrm{x}$ is the number of nuclei in each individual well while $\mu$ and $\sigma$ are the mean and the standard deviation, respectively, of the total number of nuclei in each plate. A robust significance threshold $(\mathrm{Z}<$ -1.5 ) was applied as the cutoff for selection of cell-killing hits to maximally reduce the possibility of false positive identification, implying a probability of $99.93 \%$ that a certain antisense oligonucleotide miRNA inhibitor was a true hit (refer to https://www.dummies.com/education/ math/statistics/how-to-use-the-z-table/). 


\section{Antibodies and reagents}

The anti-DNA-PKcs antibody was purchased from Thermo Fisher Scientific Inc. (A303-967A, USA; 1:2000 dilution). Antibodies specific for ATR (\#2853), phosphoATR (Thr1989) (\#58014), CHK1 (\#2360), phospho-CHK1 (Ser317) (\#2344), phospho-p53 (Ser20) (\#9287), p53 (\#9282), and GAPDH (\#5174) were purchased from Cell Signaling and 1:1000 diluted for use. Antibodies specific for YY1AP1 (NBP1-81763, 1:1000 dilution), YY1 (NBP146218, 1:2000 dilution), and FEN1 (NB100-150, 1:1000 dilution) were purchased from Novus Biologicals (USA). Antibodies specific for caspase-3 (ab32351, 1:2000 dilution), cleaved-caspase-3 (ab32042, 1:500 dilution), and Bax (ab182733, 1:2000 dilution) were purchased from Abcam (USA). DNA-PKcs inhibitor: VX-984 (HY19939S) and NU-7441 (HY-11006) were purchased from MedChemExpress (USA).

\section{Luciferase plasmid construction and luciferase assay}

We constructed a luciferase-UTR reporter plasmid that contained the YYAP1 3'-UTR region carrying a wild-type or mutant miR-1193 binding site (Fig. 4a). In brief, reverse complimentary oligonucleotides for each selected region containing either a putative or mutated hsa-miR-1193 binding site in the $3^{\prime}$-UTR of YY1AP1 were hybridized to generate double-stranded DNA for insertion into the pMIRReporter ${ }^{\mathrm{TM}}$ firefly luciferase vector (Applied Biosystems, CA, USA) at the SacI and HindIII sites. All constructs were confirmed by sequencing. For the luciferase assay, $293 \mathrm{~T}$ cells were co-transfected with the appropriate plasmids and $100 \mathrm{nM}$ wild-type or mutant miR-1193 mimic in 48-well plates. Cells were harvested $48 \mathrm{~h}$ after transfection, lysed and analyzed using a luciferase assay kit (Promega, Madison, WI, USA) according to the manufacturer's instructions. $\beta$-Galactosidase was used for normalization.

\section{Quantitative RT-PCR (qRT-PCR)}

To measure miR-1193 and YY1AP1 mRNA levels, qRTPCR was performed in triplicate with TaqMan ${ }^{\circledR}$ Universal PCR Master Mix and a specific TaqMan ${ }^{\circledR}$ MicroRNA assay (Applied Biosystems) in an ABI PRISM® 7000 Sequence Detection System (Applied Biosystems). mRNA expression levels were normalized to the U6 expression level, and relative quantification was performed using the $2^{-\Delta \Delta C T}$ method. Information on primer sequences is presented in Supplemental Table 1.

\section{Cell survival assay and cell viability assay}

Cell survival was assessed by evaluating the colonyforming ability. In brief, M059J, M059K, and U251 cells were seeded in six-well plates (500 cells per well) after transfection with anti-miR-1193 or anti-miR-NC or treatment with a DNA-PKcs inhibitor (VX-984: 0.1, 0.5, and $1 \mu \mathrm{M}$ or NU-7441: $0.5,1$, and $2 \mu \mathrm{M})$ and were subsequently incubated for 2 weeks to allow colonies to develop. To maximize the effects of the antisense miRNAs, the second transfection was performed one week after the first transfection. Cells were continuously exposed to DNA-PKcs inhibitors for 14 days since the day of first transfection. The medium was replaced every $72 \mathrm{~h}$ with medium containing fresh DNA-PKcs inhibitors. Cells were finally fixed with cold methanol, and the colonies were stained with crystal violet (in a $100 \%$ methanol solution) for manual counting.

The viability of M059J, M059K and U251 cells was assessed with a Cell Counting Kit-8 (CCK8) kit (Cat\#NN517, DOJINDO Laboratories, Japan) according to the manufacturer's instructions. Cells were seeded in 96-well plates and cultured in a $37^{\circ} \mathrm{C}$ incubator for up to 4 days, and the OD at $450 \mathrm{~nm}$ was measured. All cellbased assays were performed in at least triplicate.

\section{Immunofluorescence staining}

M059J and M059K cells were cultured in six-well plates and transfected with anti-miR-1193 or anti-miR-NC. After 3 days of incubation, cells were washed with PBS and fixed with $4 \%$ formaldehyde. Cells were permeabilized with Triton X-100 (0.05\%) for 10 min, blocked with 3\% BSA in PBS and then incubated overnight at $4{ }^{\circ} \mathrm{C}$ with primary antibodies (anti-53BP1, Abcam, ab175933, 1:200 dilution; anti-RPA, Abcam, ab2175, 1: 200 dilution; and anti- $\gamma$ H2AX, Cell Signaling, \#2577, 1:800 dilution). Next, cells were washed and incubated with the corresponding AF488- or AF647-conjugated secondary antibody. Finally, cells were washed with PBST and stained with DAPI for 10 min at RT. Images of the mounted slides were acquired with a Zeiss Axiovert $200 \mathrm{M}$ microscope.

\section{TUNEL assay}

M059J and M059K cells were cultured in six-well plates and transfected with anti-miR-1193 or anti-miR-NC for 4 days as described above. Cells were then washed with PBS, fixed with $4 \%$ formaldehyde in PBS for $30 \mathrm{~min}$ and washed with PBS again. Triton X-100 (1\%) and 3\% $\mathrm{H}_{2} \mathrm{O}_{2}$ were applied to the cells for $5 \mathrm{~min}$ and $10 \mathrm{~min}$, respectively. Next, cells were washed twice with ice-cold PBS, incubated with $\mathrm{TdT}$ labeling solution at $37^{\circ} \mathrm{C}$ for $1 \mathrm{~h}$, and gently washed with PBS three times. Finally, cells were incubated with $100 \mu \mathrm{l}$ staining buffer for $30 \mathrm{~min}$ in the dark, washed with PBS and stained with DAPI for imaging.

\section{Assessment of metaphase spread and nuclear morphology}

The chromosome breakage assay was performed as described previously ${ }^{34}$. In brief, M059J and M059K cells were transfected with anti-miR-1193 or anti-miR-NC. After 4 days of culture, cells were treated with $0.5 \%$ 
colchicine for $4 \mathrm{~h}$ to induce metaphase arrest, and were incubated with hypotonic solution $(0.56 \% \mathrm{KCl})$ at room temperature for $30 \mathrm{~min}$ and then in a $37{ }^{\circ} \mathrm{C}$ water bath for 5 min. Fixation with precooled fixation buffer (ethanol: methanol $=1: 3$ ) was repeated three times, and a dropper was used to place cells onto a clean slide. Spread cells were incubated at $55{ }^{\circ} \mathrm{C}$ overnight and stained with Giemsa solution (GS-500, Sigma) for image acquisition of aberrant chromosomes with a Zeiss Axiovert $200 \mathrm{M}$ microscope.

\section{Flow cytometric apoptosis assay}

The flow cytometric apoptosis assay was carried out with an Annexin-V FITC/PI staining kit (ab14085, Abcam, UK) according to the manufacturer's instructions. In brief, $2 \times 10^{5}$ M059K or M059J cells per well were seeded into six-well plates and allowed to attach overnight. Cells were then transfected with anti-miR-1193 or anti-miR-NC and incubated for $96 \mathrm{~h}$ in a humidified $\mathrm{CO}_{2}$ incubator. Subsequently, cells were trypsinized and centrifuged at $1000 \mathrm{rpm}$ for $5 \mathrm{~min}$. The obtained cell pellet was washed with DPBS and stained with both Annexin-V FITC and PI for $15 \mathrm{~min}$. The stained cells were washed again with DPBS to remove excess dye and were finally resuspended in $300 \mu \mathrm{l}$ of $1 \times$ PBS. Apoptotic events were analyzed, and untreated cells were used as the negative control for gating. A total of 10,000 events were recorded during the experiment. The percentages of live, early apoptotic, late apoptotic, and necrotic cells were analyzed with a BD FACSCalibur device and analyzed with FCS express V3 (BD Biosciences, USA).

\section{EdU FACS analysis}

The S-phase analysis was carried out by flow cytometry (BD FACSCanto ${ }^{\mathrm{TM}}$ II, BD Bioscience, USA) using EdU staining assay according to the manufacturer's instructions. In brief, $2 \times 10^{5}$ M059K or M059J cells per well were seeded into six-well plates and allowed to attach overnight. Cells were then transfected with anti-miR-1193 or anti-miR-NC, fixed with $4 \%$ formaldehyde in PBS, washed with $1 \%$ BSA and incubated for 30 min with ClickiT EdU reaction solution. After incubation, samples were washed, resuspended in $1 \% \mathrm{BSA}$, and analyzed with a BD FACSCalibur device and analyzed with FCS express V3 (BD Biosciences, USA).

\section{Western blot analysis}

Cells subjected to different treatments were harvested and lysed in lysis buffer $(50 \mathrm{mM}$ Tris- $\mathrm{HCl}(\mathrm{pH} 7.4)$, $0.15 \mathrm{M} \mathrm{NaCl}$, and $1 \%$ Triton X-100 in PBS, supplemented with protease and phosphatase inhibitors) on ice for $30 \mathrm{~min}$. Proteins were separated by SDS-PAGE on an 8-16\% gel (Invitrogen) and transferred to a PVDF membrane. After blocking, the membrane was incubated with primary antibodies (described in the 'Antibodies and reagents' sections) and the corresponding secondary antibodies. Immunoreactions were visualized using ECL western blot detection reagents (Pierce Biotechnology) and Image Lab 5.1 gel densitometry analysis system. ImageJ software (version 1.8.0.) was used to analyze protein bands.

\section{Statistical analysis}

All experimental data are expressed as the mean and standard deviation (mean $\pm \mathrm{SD}$ ) values. Statistical analysis was performed using Student's independent $t$-test, and two-sided $p$-values were calculated via GraphPad Prism 8.4.2 software to assess the significance of differences between experimental groups. A $p$-value of $<0.05$ was considered to indicate a significant difference.

\section{Results}

Identification of miR-1193 as a synthetic lethal partner of DNA-PKcs by high-throughput screening

Many miRNAs are related to cancer cell proliferation, development, and migration. To discover effective miRNA partners with synthetic lethality phenotypes in DNA-PKcs-deficient GBM cells, an automated, miniaturized screening approach was established to minimize human interference and systematic errors. We performed a series of high-throughput screens in seven 384-well plates containing cells from two GBM cell lines-the DNA-PKcs proficient U118MG cell line and the DNAPKcs-deficient M059J cell line-transfected with a library of 2590 in-house-designed antisense oligonucleotides with reverse complementary sequences of the genomewide human miRNAs (Fig. 1a). Three days post transfection, the number of nuclei in each well was counted using an acumen ${ }^{\circledR}$ Cellista laser scanning imaging cytometer.

Data were obtained from replicate $(n=3)$ screens for computation of $\mathrm{Z}$ scores to estimate the significance of the effect of miRNA knockdown on cell growth. The screen identified miR-1193 as a potential miRNA partner with synthetic lethality in DNA-PKcs-deficient M059J cells $(Z$ score $=-1.75)$ compared with DNA-PKcsproficient U118MG cells $(\mathrm{Z}$ score $=-0.3)$ (Fig. 1b). To validate the specificity of the synthetic lethal interaction between anti-miR-1193 and DNA-PKcs deficiency, antimiR-1193 was transfected into several different human cell lines, including hepatocarcinoma cell lines (HepG2 and Huh7), a lung cancer cell line (A549), a noncancerous cell line (epithelial RPE-1) and fibroblasts. Imaging for nuclei counting (Fig. 1c) and survival assays (Fig. 1d) revealed the sensitivity to anti-miR-1193 transfection in M059J cells but not in U118MG or the other cell lines tested in this study. 

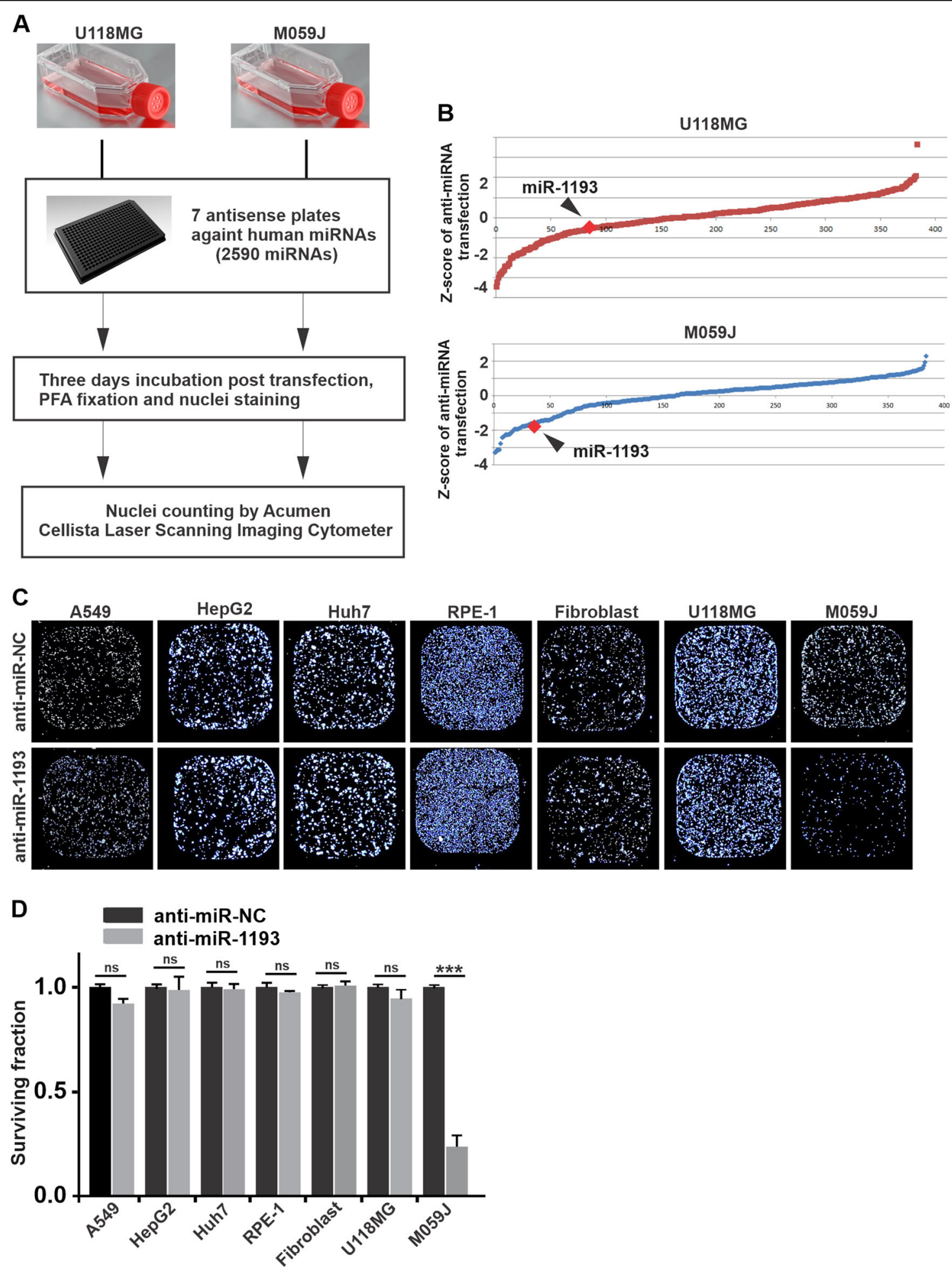

Fig. 1 miR-1193 was identified from an automated, high-throughput screen as a specific synthetic lethal interacting partner with DNAPKcs in M059J cells. a Workflow of the anti-miRNA screening procedure. GBM cells (M059J and U118MG) were seeded into seven 384-well plates in $50 \mu \mathrm{l}$ of medium on the first day. In-house designed antisense oligonucleotides targeting 2590 human miRNAs were individually transfected to knockdown the corresponding miRNAs. Cells were fixed, and nuclei were stained with Hoechst on day 4. Automated nuclei counting was performed with an acumen ${ }^{\circledR}$ Cellista laser scanning imaging cytometer. $\mathbf{b}$ Z scores were calculated from the screen of all antisense oligonucleotides targeting 2590 human miRNAs in both U118MG cells and DNA-PKcs-deficient M059J cells. The Z scores for miR-1193 are indicated in red and identified by black arrows. c Effects of anti-miR-1 193 on the proliferation of U118MG, M059J and several other cell lines. $\mathbf{d}$ The survival fractions were calculated. The data are presented as the mean \pm SD values from triplicate biological experiments. ${ }^{*} P<0.05,{ }^{* *} P<0.01,{ }^{* *} P<0.005$. NS not significant: $p>0.05$. 
Validation of miR-1193/DNA-PKcs synthetic lethality

To further demonstrate the specific synthetic lethal interaction in GBM cells, we treated M059K and U251 cells with two highly specific DNA-PKcs inhibitors, VX-984 $4^{35}$, and NU-7441 ${ }^{36}$, upon the transfection with anti-miR-1193. Similarly, as shown by the results of the clonogenic survival (Fig. 2a-f) and viability assays (Fig. $2 \mathrm{~g}-\mathrm{j}$ ), cell proliferation and cell survival were suppressed in a dose-dependent manner when both DNA-PKcs and miR-1193 were inhibited. Collectively, these data suggest that anti-miR-1193 is a specific synthetic lethal partner sensitive to GBM cells deficient in DNA-PKcs.

We also confirmed the synthetic lethality of anti-miR1193 treatment/DNA-PKcs deficiency using some other approaches in M059J and M059K cells with the same origin and thus the same genetic background. The expression levels of miR-1193 and DNA-PKcs in M059J and M059K cells after miR-1193 expression was inhibited by antisense oligonucleotide transfection demonstrated effective inhibition of miR-1193 expression in both cell lines (Fig. S1a) but deficiency of DNA-PKcs only in M059J cells (Fig. S1b). In the clonogenic survival assays, miR1193 inhibition significantly impaired the survival of DNA-PKcs-deficient M059J cells compared with DNAPKcs-proficient M059K cells (Fig. 3a, b). In addition, significant synthetic lethal inhibitory effects of anti-miR1193 in DNA-PKcs-deficient cells were observed $96 \mathrm{~h}$ post transfection by a CCK8 cell viability assay (Fig. 3c). The TUNEL assay results demonstrated much more significant apoptotic DNA fragmentation in M059J cells due to knockdown of miR-1193 than in M059J cells (Fig. 3d, e), compared with nontargeting knockdown or in M059K cells with inhibition of miR-1193. Similar results were obtained with Annexin-V/PI staining (Fig. 3f, g). Cell death related proteins were also assayed, with the level of cleaved-caspase-3 and Bax significantly enhanced in M059J cells with miR-1193 deficiency (Fig. 3h). Collectively, our data suggest specific synthetic lethality of miR1193 inhibition with DNA-PKcs deficiency in M059J cells.

\section{YY1AP1 is a direct downstream target of miR-1193}

Identification of miR-1193 targets is critical for understanding the biological functions of this miRNA in mediating synthetic lethality in M059J cells. Therefore, we compiled two lists of candidate target genes for miR-1193 (data not shown) from two well-recognized prediction databases, i.e., miRDB (version 4.0) and TargetScan (release 6.2) and found that YY1AP1 was one of the top candidate genes in both lists. Our previous study showed that $Y Y 1$ can downregulate FEN1 expression by directly binding to the FEN1 coding sequence ${ }^{37}$. FEN1 plays essential roles in repair of DNA DSB damage and thus promotes tumor cell survival ${ }^{38}$, furthermore, YY1AP1 is related to transcriptional regulation, DNA repair and replication presumably through interacting with $Y Y 1^{39}$. Thus, we focused on YY1AP1 as the candidate target gene of miR-1193 for further investigations in this study and reasoned that YY1AP1 might regulate the expression of both $F E N 1$ and $Y Y 1$. To validate the direct regulation of FEN1 by YY1AP1/YY1, the expression levels of YY1 and FEN1 were measured after YY1AP1 was overexpressed or ablated in $293 \mathrm{~T}$ cells. FEN1 expression was clearly suppressed but YY1 expression was enhanced by transfection of the YY1AP1 expression plasmid (ORF YY1AP1). On the other hand, when YY1AP1 was downregulated by YY1AP1 siRNA (siYY1AP1), FEN1 expression was enhanced, but YY1 expression was suppressed. Taken together, these results indicate that as a YY1-associated protein, YY1AP1 can negatively regulate FEN1 expression by interacting with YY1 (Fig. S2a).

Moreover, we found that the $3^{\prime}$-UTR of YY1AP1 contains a precise miR-1193 binding site (Fig. 4a). We sought to verify that YY1AP1 is a direct target gene of miR-1193. To this end, we conducted a luciferase reporter assay to determine whether the putative binding site of miR-1193 in the 3'-UTR of YY1AP1 is required for miR-1193regulated gene translation. MiR-1193 repressed the activity of the luciferase reporter containing the wild-type YY1AP1-3'-UTR but barely affected the activity of the reporter containing the mutated miR-1193 binding site (Fig. 4b). Furthermore, YY1AP1 mRNA expression was significantly downregulated (Fig. 4d) upon anti-miR-1193 transfection (Fig. 4c) in both M059J and M059K cells.

\section{DNA-PKcs-deficient cells are more sensitized to anti-miR-} 1193 treatment through the YY1AP1/YY1/FEN1 pathway

To examine the effect of anti-miR-1193 on the YY1AP1/YY1/FEN1 axis in DNA-PKcs null cells, we measured the protein expression levels of YY1AP1, YY1, and FEN1 after anti-miR-1193 transfection. As expected, compared with anti-miR-NC transfection, anti-miR-1193 transfection increased the endogenous protein levels of YY1AP1 and YY1 in both M059J and M059K cells, while FEN1 expression was abrogated by anti-miR-1193 transfection (Fig. 4e). These results indicate that YY1AP1 expression is directly regulated by miR-1193 via seedmatching sequences.

To evaluate the necessity of FEN1 for GBM cell proliferation, cell survival assays were performed after FEN1 was downregulated by shFEN1 transfection. Significant inhibition of colony formation, cell growth, and cell viability were observed in DNA-PKcs-deficient M059J cells transfected with shFEN1 (Fig. 4f, g). In addition, FEN1 depletion led to decreased proliferation, as assessed by the EdU assay from Fluorescence activated cell scanning (FACS) and immunofluorescence staining (Figs. 4h, S2b, c), and to increased apoptosis, as assessed by the TUNEL assay (Fig. 4i, j), of M059J cells. The evidence presented 


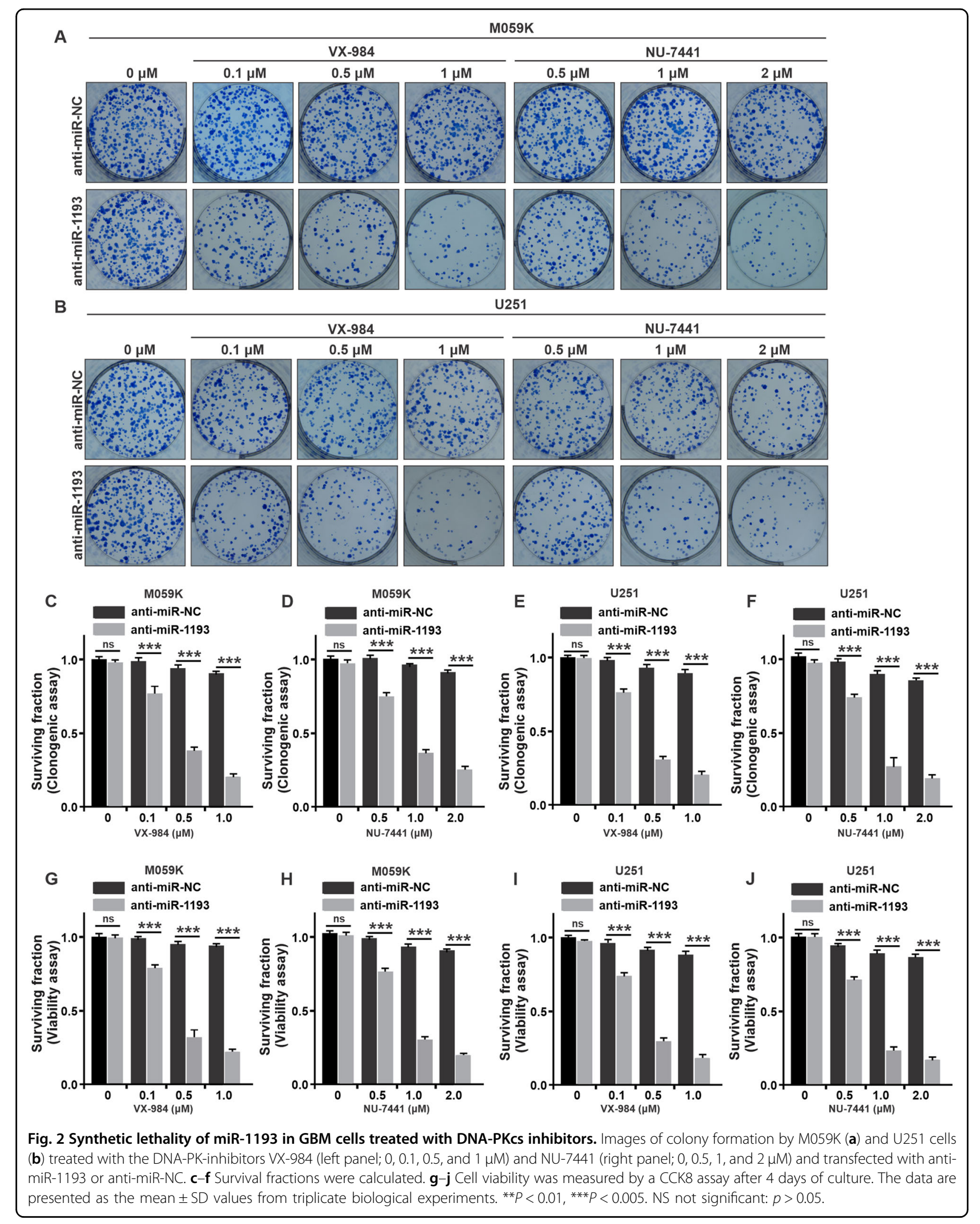




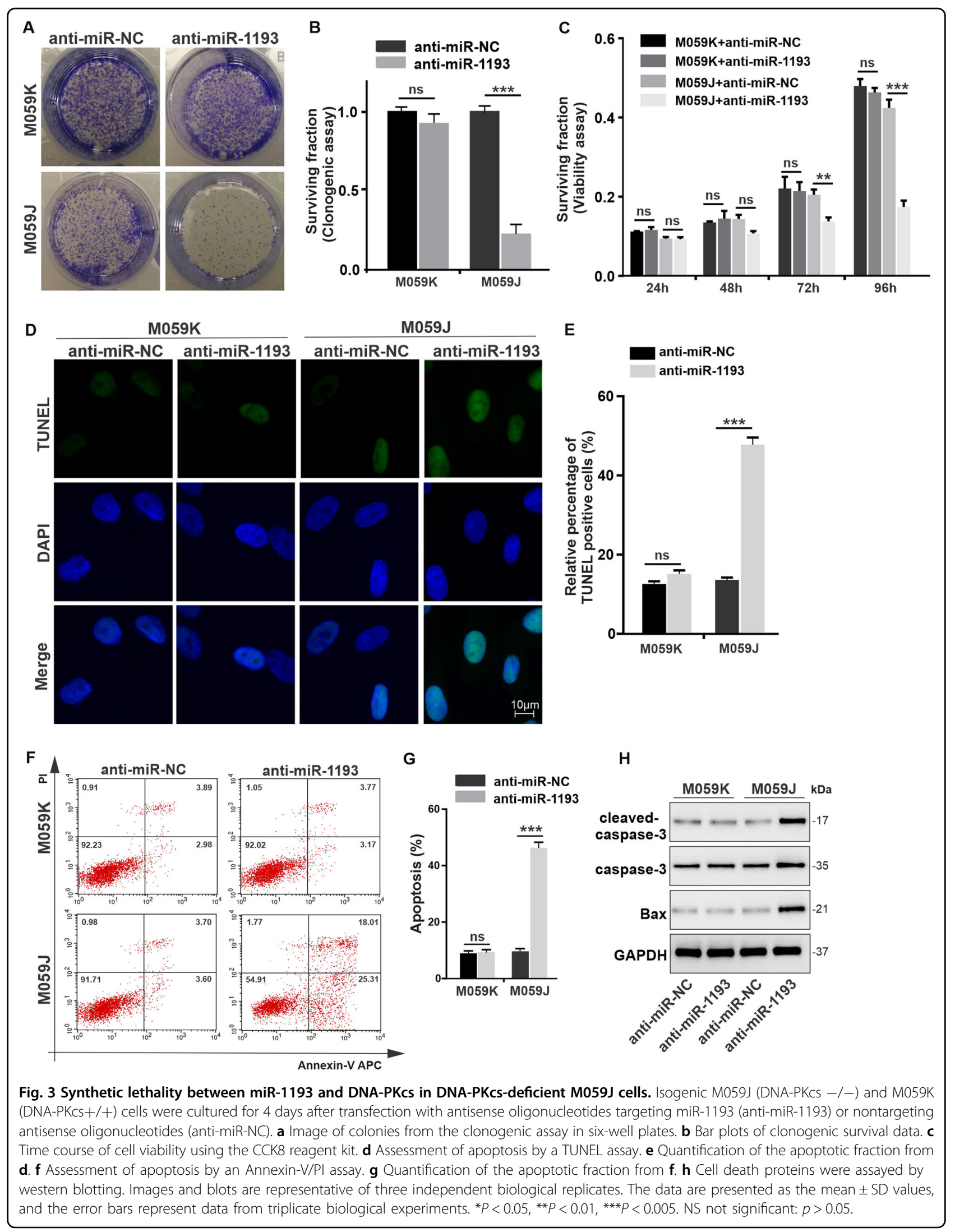




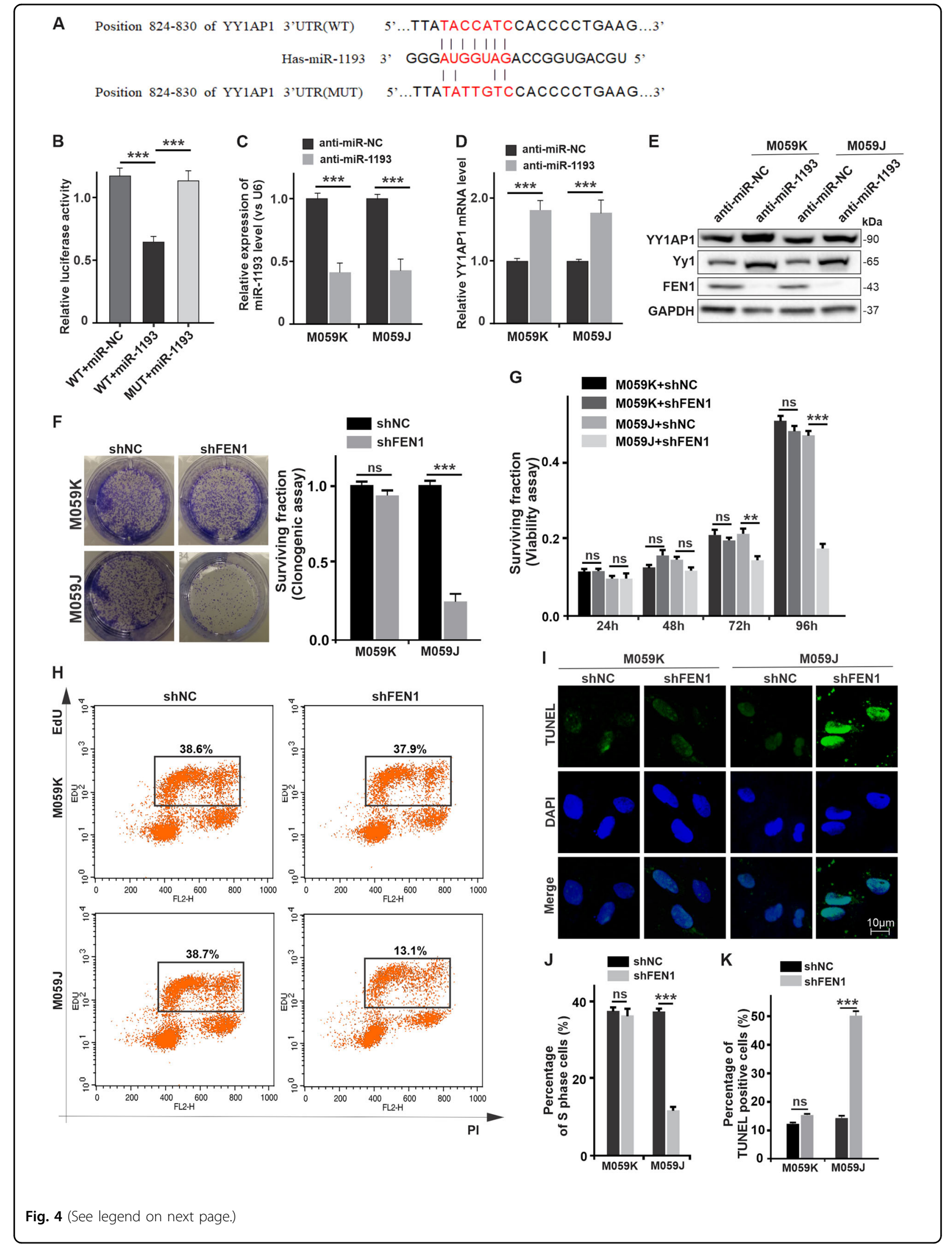


(see figure on previous page)

Fig. 4 miR-1193 directly targets YY1AP1 and exhibits synthetic lethality with DNA-PKcs through the YY1-FEN1 pathway. a Predicted miR1193 binding sequence in the 3'-UTR of YY1AP1 (wild-type YY1AP1-3'-UTR) and in a mutant containing seven altered nucleotides indicated in red (YY1AP1-3'-UTR-mut). b Luciferase activity of the reporter constructs containing the 3'UTR of YY1AP1 with the wild-type or mutated miR-1193 binding site in 293 T cells after co-transfection with the control or miR-1193 expression constructs. The mRNA levels of miR-1193 (c) and YY1AP1 (d) were measured by RT-qPCR in M059K or M059J cells transfected with anti-miR-NC or anti-miR-1193. U6 RNA was used as the internal control. e Protein expression levels of YY1AP1, YY1, and FEN1 in M059K and M059J cells transfected with anti-miR-NC or anti-miR-1193. GAPDH was used as the loading control. f Colony formation assay of M059K and M059J cells transfected with shNC and shFEN1 and cultured for 4 days. The expression levels of FEN1 and GAPDH are presented. $\mathbf{g}$ The viability of transfected M059K and M059J cells was measured with a CCK8 kit across a time course. $\mathbf{h}$ Cell proliferation was measured by an EdU analysis of FACS. i Apoptosis was measured by a TUNEL assay and quantified. $\mathbf{j}$, $\mathbf{k}$ The percentage of cells in Sphase and apoptosis under each condition was quantified. The images are representative of three independent biological replicates. The data are presented as the mean \pm SD values, and the error bars represent data from triplicate biological experiments. ${ }^{*} P<0.05,{ }^{* *} P<0.01,{ }^{* * *} P<0.005$. NS not significant: $p>0.05$.

here reveals that the synthetic lethal interaction between miR-1193 inhibition and DNA-PKcs deficiency significantly suppresses cell growth and promotes apoptosis through the YY1AP1/YY1/FEN1 pathway in M059J cancer cells.

To further confirm the synthetic lethal interaction between anti-miR-1193 and DNA-PKcs deficiency, we depleted both miR-1193 and DNA-PKcs in M059K cells by simultaneous transfection of anti-miR-1193 and DNAPKcs-targeted siRNA. The results of the clonogenic survival assay (Fig. S3a) showed that, similar to the effects in M059J cells transfected with anti-miR-1193, co-depletion of miR-1193 and DNA-PKcs significantly inhibited the proliferation of M059K cells. Interestingly, when DNAPKcs was expressed in M059J cells by transfecting the overexpression plasmid ORF DNA-PKcs, the miR-1193 deficiency-induced inhibition of proliferation was reversed (Fig. S3b-e). The TUNEL assay demonstrated a significant enhancement in apoptosis in M059K cells transfected with siDNA-PKcs and a reduction in apoptosis in M059J cells co-transfected with anti-miR-1193 and ORF DNA-PKcs (Fig. S3f, S3g). These data further confirm the synthetic lethal interaction between antimiR-1193 and DNA-PKcs deficiency.

\section{Anti-miR-1193 increases DSB damage in M059J cells with DNA-PKcs deficiency}

In addition to participating in the LP-BER pathway ${ }^{40}$, FEN1 is also an important regulator of HR and MMEJmediated DSB repair ${ }^{41,42}$, suggesting an alternative mechanism by which the DSB repair pathway promotes the survival of DNA-PKcs-deficient M059J cells. In this scenario, DSBs might not be repaired in DNA-PKcsdeficient cells upon depletion of miR-1193/FEN1. To test this hypothesis, an immunofluorescence technique was applied to monitor the presence of $\gamma \mathrm{H} 2 \mathrm{AX}$ and 53BP1 nuclear foci, markers of DNA DSB damage ${ }^{43}$, in M059K cells and M059J cells transfected with anti-miR-1193 or anti-miR-NC. Anti-miR-1193 induced the expression of $\gamma \mathrm{H} 2 \mathrm{AX}$, and 53BP1 expression was more pronounced in DNA-PKcs-deficient M059J cells (Fig. 5a, c, and d). A similar induction of $\gamma \mathrm{H} 2 \mathrm{AX}$ and 53BP1 expression was detected in M059J cells transfected with shFEN1 (Fig. S4a, S4b, and S4c). Taken together, these observations suggest a DSB repair defect in GBM cells lacking both miR-1193 and DNA-PKcs activity.

To explore the blockade of DSB repair in miR-1193/ FEN1-deficient cells, we investigated the ssDNA repair intermediates generated by DNA end resection during the induction of pre-apoptosis in miR-1193/FEN1-depleted cells. The presence of nuclear RPA foci, a marker of ssDNA repair intermediates ${ }^{44}$, was assessed by an immunofluorescence assay in both M059J and M059K cells transfected with anti-miR-1193 or anti-miR-NC. In contrast to M059K cells, M059J cells transfected with anti-miR-1193 exhibited compelling accumulation of RPA foci (Fig. 5b, e), consistent with the low levels of $\gamma \mathrm{H} 2 \mathrm{AX}$ and 53BP1 foci (Fig. 4a). Similar results for RPA foci were observed in M059J cells transfected with shFEN1 (Fig. S4d and S4e). Collectively, these data indicate that DSBs persist in DNA-PKcs-deficient cells and yield RPA-coated ssDNA structures upon miR-1193/FEN1 knockdown, promoting the induction of the preapoptotic pathway.

\section{miR-1193/DNA-PKcs deficiency activates the ATR/Chk1/ p53 apoptosis axis}

Subsequently, we sought to determine whether DSB deficiency and ssDNA repair intermediates induce apoptotic signaling in anti-miR-1193-treated/DNA-PKcs-deficient cells. As the expression level of ATM is low in M059 cells $^{10}$, we reasoned that the apoptosis induced by anti-miR-1193 treatment/DNA-PKcs deficiency might be dependent on ataxia-telangiectasia mutated and Rad3related protein kinase (ATR). ATR is a critical component of the cellular DDR, which is activated by DNA damage and replication stress ${ }^{45}$. To test this hypothesis, we assessed the activation status of the ATR/Chk1/ p53 signaling axis in M059J and M059K cells transfected 
with anti-miR-1193 or anti-miR-NC by immunoblotting with antibodies against phospho-ATR $\left(\mathrm{Thr}^{1989}\right.$ ), phosphoChk1 $\left(\operatorname{Ser}^{317}\right)$, total p53 and phospho-p53 $\left(\operatorname{Ser}^{20}\right)$. Using these assays, we found the ATR/Chk1/p53 axis to be activated in the DNA-PKcs-defective M059J cells upon miR-1193 inhibition (Fig. 6a). The data in Fig. 6a demonstrate the activation of the ATR/Chk1/p53 apoptosis signaling pathway in miR-1193/DNA-PKcs-deficient M059J cells, suggesting that miR-1193/YY1AP1/YY1/ FEN1 inhibition leads to ATR/Chk1-dependent p53 activation in DNA-PKcs-deficient cells.
Genomic instability is enhanced in M059J cells with miR1193/DNA-PKcs deficiency

Finally, we observed that inhibition of miR-1193 in DNA-PKcs-deficient M059J cells increased the frequency of chromosomal aberrations compared with that in wildtype M059K cells (Fig. 6b, c). In the nuclear morphology assay, miR-1193 depletion in M059J cells increased the nuclear heteromorphism, with budding cells, micronuclei, and multinucleated giant cells (Fig. 6d, e). Collectively, these data indicated that chromatin instability is significantly increased by accumulated DSB damage and

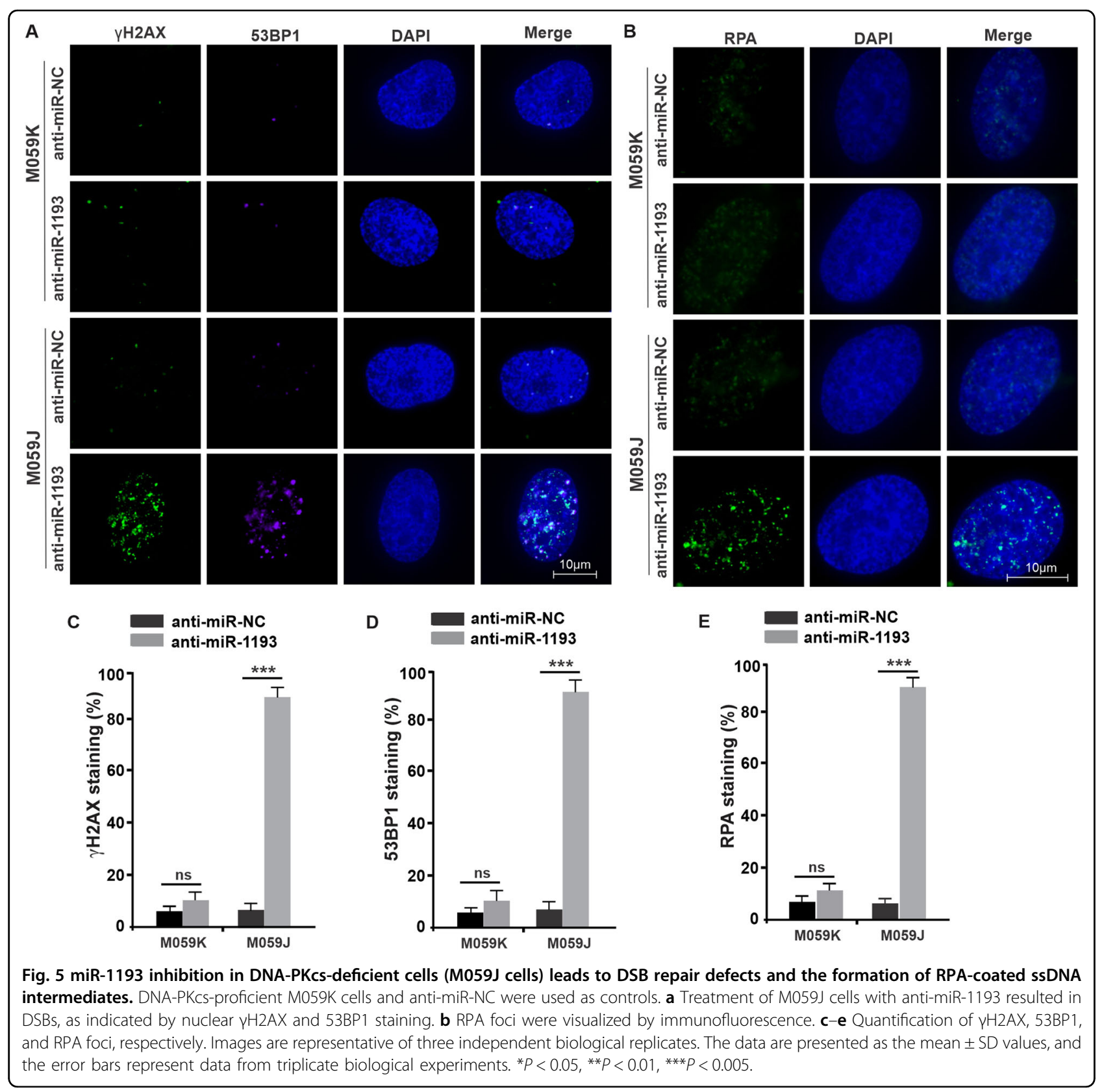




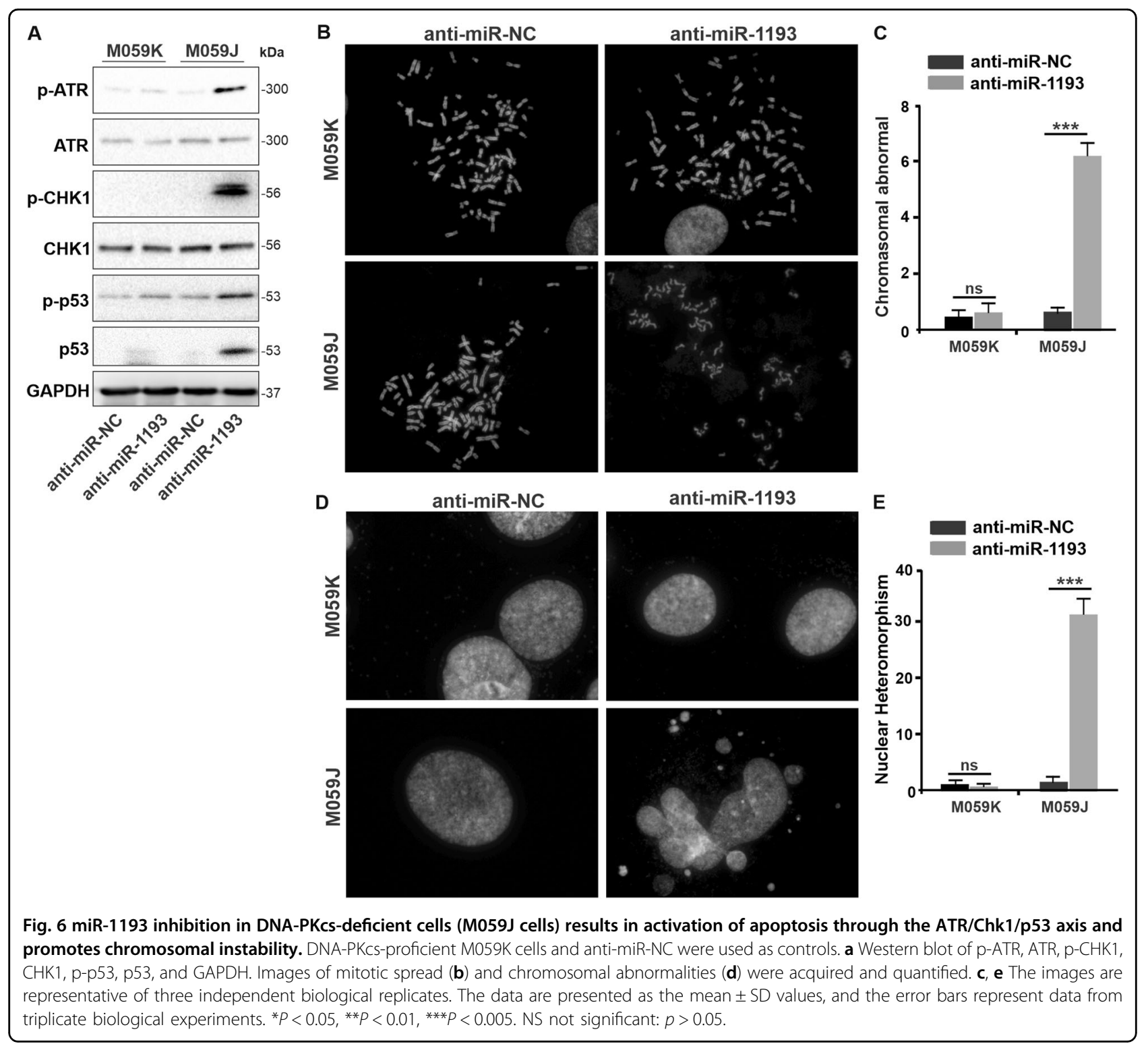

apoptosis activation due to the synthetic lethal interaction between anti-miR-1193 treatment and DNA-PKcs deficiency in GBM cells.

\section{Discussion}

GBM remains the most frequent malignant primary brain tumor, with the highest mortality rate among cancers and very few effective therapies ${ }^{46}$. Synthetic lethality is increasingly recognized as an essential treatment option, especially for cancers harboring certain gene mutations ${ }^{47}$. The flexibility of using miRNA inhibition in synthetic lethal therapies predicts a new era in cancer treatment $^{25,48}$. However, very few synthetic lethal interaction partners have been identified or tested in clinical trials. In this study, we performed a highly efficient automated, high-throughput screen, which identified miR-1193 as a potential anticancer therapeutic candidate for DNA-PKcs-deficient cancers. Based on the robust data shown above, we proposed a working model (Fig. 7) to clarify the mechanism underlying the synthetic lethality between DNA-PKcs deficiency and miR-1193 inhibition. Perturbation of either DNA-PKcs or miR-1193 alone did not decrease the viability of cancer cells (Fig. 7a). The miR-1193/YY1AP1/YY1/FEN1 axis regulates $H R$ and MMEJ-mediated DSB repair, providing a compensating pathway in DNA-PKcs-positive cells in which NHEJmediated DSB repair is active. Therefore, perturbation of both DSB repair pathways (HR/MMEJ and NHEJ) by simultaneous inhibition of miR-1193 and ablation of DNA-PKcs can lead to marked loss of viability (Fig. 7b). 


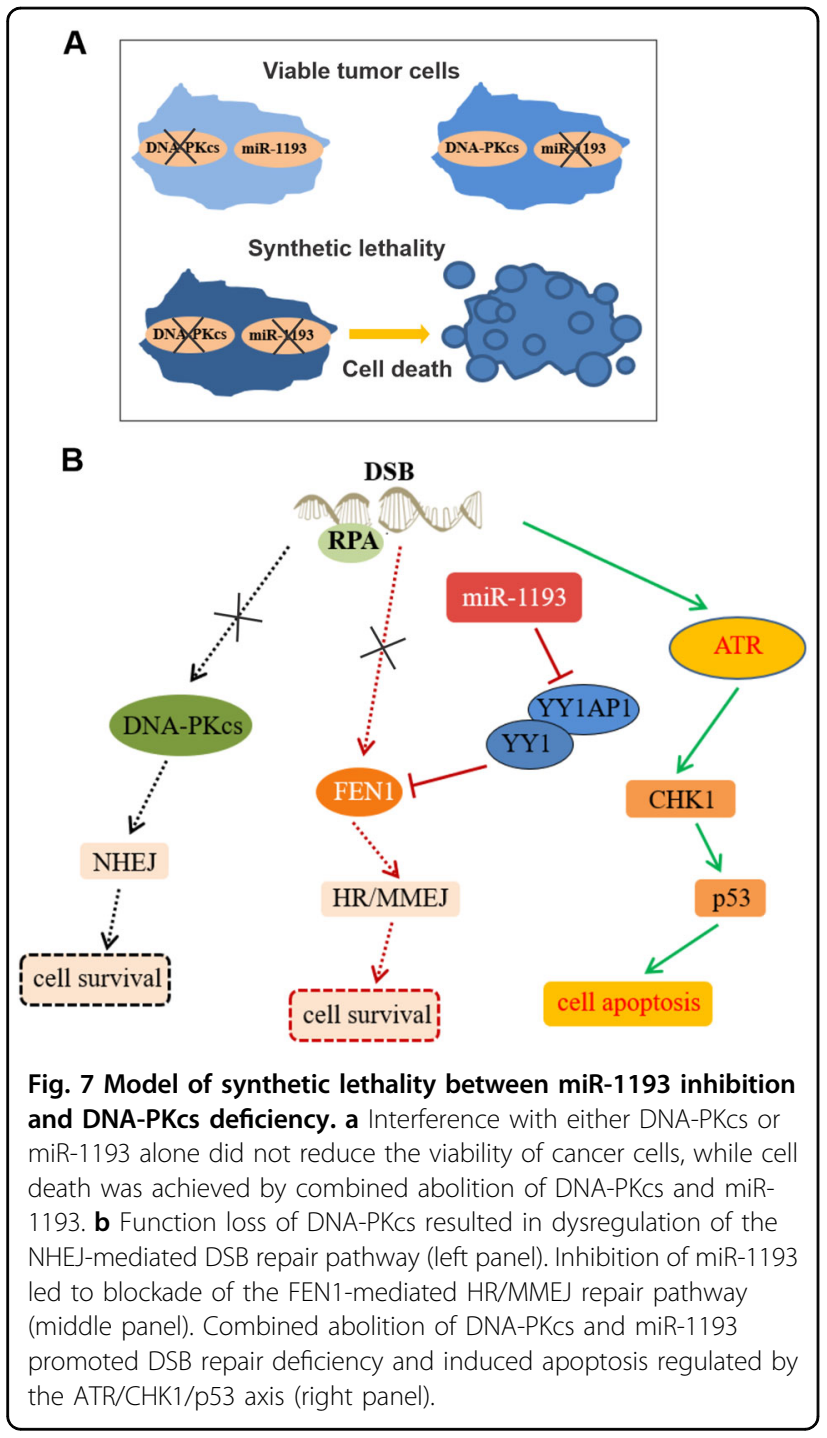

Simultaneous elimination of these different DSB repair pathways leads to activation of the ATR/CHK1/p53 axis and subsequently promotes tumor cell apoptosis (Fig. 7b).

Our data suggest that anti-miR-1193 could potentially be applied as a single-agent treatment for DNA-PKcsdeficient GBM tumors. The highly malignant nature of human GBMs with DNA-PKcs deficiency and the possibility of inducing synthetic lethality via miR-1193 inhibition provide insights for biomarker-driven animal or clinical trials. In addition to our findings in this study, accumulating data suggest that miRNAs are a valuable means to achieve synthetic lethality in certain tumors with specific genetic deficiencies ${ }^{49,50}$. However, this promising approach still has limitations, among which are potential off-target effects. Chemical modifications of antisense oligonucleotides targeting miRNAs, such as $2^{\prime}$-O-methyl modification, have been shown to minimize the off-target effects and could dramatically increase the in vivo delivery efficiency $^{51}$. A thiol- and cholesterol-conjugated 2'-Omethyl-modified antagomir might be implemented to further validate miR-1193 as a promising therapeutic target for DNA-PKcs-deficient GBM tumors. With the high in vivo delivery efficiency of chemically modified antisense oligonucleotides targeting miRNAs, trials could be conducted in GBM, which exhibits a high frequency of DNA-PKcs deficiency and has a limited standard-of-care therapeutic response and few targeted therapeutic approaches.

In conclusion, this study highlights the importance of the miR-1193/YY1AP1/YY1/FEN1 axis as a promising therapeutic target for DNA-PKcs-deficient GBM tumors, with synthetic lethality as the mechanism of action. The evidence generated in this study provides a novel and promising therapeutic intervention strategy for DNAPKcs-deficient GBM, and further in-depth investigations are needed.

\section{Acknowledgements}

This project was founded by the National Natural Science Foundation (Grant No. 31701179), National Natural Science Foundation of China (Grant Nos. 81872284), the Natural Science Foundation of Jiangsu Province (Grant No. BE2018714), and the China Postdoctoral Science Foundation (Grant No. 2016M591877).

\section{Author details}

${ }^{1}$ Jiangsu Key Laboratory for Molecular and Medical Biotechnology, College of Life Sciences, Nanjing Normal University, 210097 Nanjing, Jiangsu, P.R. China. ${ }^{2}$ Key Laboratory for Molecular Biotechnology, College of Life Sciences, Nanjing University, 210093 Nanjing, Jiangsu, P.R. China. ${ }^{3}$ Department of Neurosurgery, The First Affiliated Hospital of Nanchang University, 330006 Nanchang, R.P. China. ${ }^{4}$ Department of Oncology-Pathology, Karolinska Institute, Stockholm 17176, Sweden

\section{Author contributions}

J.Z., J.P.L., and Z.G.G. designed and supervised this study, analyzed the data, and co-wrote the paper. J.Z., L.J., S.T., Z.G.H., L.F.H., Y.B.L., and E.M.Z. performed the experiments and analyzed the data. All authors read and approved the manuscript.

\section{Conflict of interest}

The authors declare that they have no conflict of interest.

\section{Publisher's note}

Springer Nature remains neutral with regard to jurisdictional claims in published maps and institutional affiliations.

Supplementary Information accompanies this paper at (https://doi.org/ 10.1038/s41419-020-02812-3).

Received: 3 February 2020 Revised: 20 July 2020 Accepted: 20 July 2020 Published online: 30 July 2020

\section{References}

1. Stupp, R. et al. Effects of radiotherapy with concomitant and adjuvant temozolomide versus radiotherapy alone on survival in glioblastoma in a randomised phase III study: 5-year analysis of the EORTC-NCIC trial. Lancet Oncol. 10, 459-466 (2009).

2. Dunn, G. P. et al. Emerging insights into the molecular and cellular basis of glioblastoma. Genes Dev. 26, 756-784 (2012). 
3. Harper, J. W. \& Elledge, S. J. The DNA damage response: ten years after. Mol. Cell 28, 739-745 (2007).

4. Reinhardt, H. C. \& Yaffe, M. B. Kinases that control the cell cycle in response to DNA damage: Chk1, Chk2, and MK2. Curr. Opin. Cell Biol. 21, 245-255 (2009).

5. Lees-Miller, S. P. et al. Absence of p350 subunit of DNA-activated protein kinase from a radiosensitive human cell line. Science 267, 1183-1185 (1995).

6. Truong, L. N. et al. Homologous recombination is a primary pathway to repair DNA double-strand breaks generated during DNA rereplication. J. Biol. Chem. 289, 28910-28923 (2014).

7. Truong, L. N. et al. Microhomology-mediated end joining and homologous recombination share the initial end resection step to repair DNA doublestrand breaks in mammalian cells. Proc. Natl Acad. Sci. USA 110, 7720-7725 (2013).

8. Jin, M. H. \& Oh, D. Y. ATM in DNA repair in cancer. Pharm. Ther. 203, 107391 (2019).

9. Ceccaldi, R., Rondinelli, B. \& D'Andrea, A. D. Repair pathway choices and consequences at the double-strand break. Trends Cell Biol. 26, 52-64 (2016).

10. Ng, W. L., Yan, D., Zhang, X., Mo, Y. Y. \& Wang, Y. Over-expression of miR-100 is responsible for the low-expression of ATM in the human glioma cell line: M059J. DNA Repair (Amst.) 9, 1170-1175 (2010).

11. Riabinska, A. et al. Therapeutic targeting of a robust non-oncogene addiction to PRKDC in ATM-defective tumors. Sci. Transl. Med. 5, 189ra178 (2013).

12. Nijman, S. M. \& Friend, S. H. Cancer. Potential of the synthetic lethality principle. Science 342, 809-811 (2013).

13. Padeken, J. et al. Synergistic lethality between BRCA1 and H3K9me2 loss reflects satellite derepression. Genes Dev. 33, 436-451 (2019).

14. Sanjiv, K. et al. Cancer-specific synthetic lethality between ATR and CHK1 kinase activities. Cell Rep. 17, 3407-3416 (2016).

15. Lord, C. J. \& Ashworth, A. PARP inhibitors: synthetic lethality in the clinic. Science 355, 1152-1158 (2017).

16. O'Neil, N. J., Bailey, M. L. \& Hieter, P. Synthetic lethality and cancer. Nat. Rev. Genet. 18, 613-623 (2017).

17. Ryan, C. J., Bajrami, I. \& Lord, C. J. Synthetic lethality and cancer-penetrance as the Major Barrier. Trends Cancer 4, 671-683 (2018).

18. Alcaraz-Sanabria, A. et al. Synthetic lethality interaction between aurora kinases and CHEK1 inhibitors in ovarian cancer. Mol. Cancer Ther. 16, 2552-2562 (2017).

19. Sun, C. et al. BRD4 inhibition is synthetic lethal with PARP inhibitors through the induction of homologous recombination deficiency. Cancer Cell $\mathbf{3 3}$ 401-416. e408 (2018).

20. He, L. \& Hannon, G. J. MicroRNAs: small RNAs with a big role in gene regulation. Nat. Rev. Genet. 5, 522-531 (2004).

21. Garofalo, M., Condorelli, G. \& Croce, C. M. MicroRNAs in diseases and drug response. Curr. Opin. Pharm. 8, 661-667 (2008).

22. Negrini, M., Ferracin, M., Sabbioni, S. \& Croce, C. M. MicroRNAs in human cancer: from research to therapy. J. Cell Sci. 120, 1833-1840 (2007).

23. $\mathrm{Xu}, \mathrm{H}$. et al. miR-29s inhibit the malignant behavior of U87MG glioblastoma cell line by targeting DNMT3A and 3B. Neurosci. Lett. 590, 40-46 (2015).

24. Sana, J., Hajduch, M., Michalek, J., Vyzula, R. \& Slaby, O. MicroRNAs and glioblastoma: roles in core signalling pathways and potential clinical implications. J. Cell Mol. Med. 15, 1636-1644 (2011).

25. Nittner, D. et al. Synthetic lethality between Rb, p53 and Dicer or miR-17-92 in retinal progenitors suppresses retinoblastoma formation. Nat. Cell Biol. 14, 958-965 (2012).

26. Han, H., Chen, Y., Cheng, L., Prochownik, E. V. \& Li, Y. microRNA-206 impairs CMyc-driven cancer in a synthetic lethal manner by directly inhibiting MAP3K13. Oncotarget 7, 16409-16419 (2016).

27. Zeng, X. et al. FEN1 mediates miR-200a methylation and promotes breast cancer cell growth via MET and EGFR signaling. FASEB J. 33, 10717-10730 (2019).
28. Mengwasser, K. E. et al. Genetic screens reveal FEN1 and APEX2 as BRCA2 synthetic lethal targets. Mol. Cell 73, 885-899. e886 (2019).

29. Guo, Z. et al. Sequential posttranslational modifications program FEN1 degradation during cell-cycle progression. Mol. Cell 47, 444-456 (2012).

30. Li, J. L. et al. FEN1 inhibitor increases sensitivity of radiotherapy in cervical cancer cells. Cancer Med. 8, 7774-7780 (2019).

31. Moazeni-Roodi, A., Ghavami, S., Ansari, H. \& Hashemi, M. Association between the flap endonuclease 1 gene polymorphisms and cancer susceptibility: An updated meta-analysis. J. Cell Biochem. 120, 13583-13597 (2019).

32. Chen, Y. D. et al. Functional FEN1 genetic variants and haplotypes are associated with glioma risk. J. Neurooncol 111, 145-151 (2013).

33. Xie, B. et al. High-throughput screening identified mitoxantrone to induce death of hepatocellular carcinoma cells with autophagy involvement. Biochem. Biophys. Res Commun. 521, 232-237 (2020).

34. van der Crabben, S. N. et al. Destabilized SMC5/6 complex leads to chromosome breakage syndrome with severe lung disease. J. Clin. Invest. 126, 2881-2892 (2016).

35. Timme, C. R. et al. The DNA-PK inhibitor VX-984 enhances the radiosensitivity of glioblastoma cells grown in vitro and as orthotopic xenografts. Mol. Cancer Ther. 17, 1207-1216 (2018).

36. Zhao, Y. et al. Preclinical evaluation of a potent novel DNA-dependent protein kinase inhibitor NU7441. Cancer Res. https://doi.org/10.1158/0008-5472 (2006).

37. Wang, J. et al. $Y Y 1$ suppresses FEN1 over-expression and drug resistance in breast cancer. BMC Cancer 15, 50 (2015).

38. Wang, M. et al. Enhanced activity of variant DNA polymerase beta (D160G) contributes to cisplatin therapy by impeding the efficiency of NER. Mol. Cancer Res. 17, 2077-2088 (2019).

39. Ho, Y. J. et al. Prognostic significance of high YY1AP1 and PCNA expression in colon adenocarcinoma. Biochem. Biophys. Res. Commun. 494, 173-180 (2017).

40. Sun, $H$. et al. The FEN1 L209P mutation interferes with long-patch base excision repair and inducecellular transformation. Oncogene 36, 194-207 (2017).

41. Sharma, S. et al. Homology and enzymatic requirements of microhomologydependent alternative end joining. Cell Death Dis. 6, e1697 (2015).

42. Nagarajan, P. et al. Roles for the Rad27 flap endonuclease in mitochondrial mutagenesis and double-strand break repair in Saccharomyces cerevisiae. Genetics 206, 843-857 (2017).

43. Lees-Miller, S. P. \& Meek, K. Repair of DNA double strand breaks by nonhomologous end joining. Biochimie 85, 1161-1173 (2003).

44. Krasikova, Y. S., Rechkunova, N. I. \& Lavrik, O. I. [Replication protein A as a major eukaryotic single-stranded DNA-binding protein and its role in DNA repair]. Mol. Biol. (Mosk.) 50, 735-750 (2016).

45. Gupta, D., Lin, B., Cowan, A. \& Heinen, C. D. ATR-Chk1 activation mitigates replication stress caused by mismatch repair-dependent processing of DNA damage. Proc. Natl Acad. Sci. USA 115, 1523-1528 (2018).

46. Cloughesy, T. F., Cavenee, W. K. \& Mischel, P. S. Glioblastoma: from molecular pathology to targeted treatment. Annu. Rev. Pathol. 9, 1-25 (2014).

47. Huang, A., Garraway, L. A., Ashworth, A. \& Weber, B. Synthetic lethality as an engine for cancer drug target discovery. Nat. Rev. Drug Discov. 19, 23-38 (2020).

48. Li, Y., Zhu, Y. \& Prochownik, E. V. MicroRNA-based screens for synthetic lethal interactions with c-Myc. RNA Dis. https://doi.org/10.14800/rd.1330 (2016).

49. Neijenhuis, S., Bajrami, I., Miller, R., Lord, C. J. \& Ashworth, A. Identification of miRNA modulators to PARP inhibitor response. DNA Repair (Amst.) 12, 394-402 (2013).

50. Plantamura, I., Cosentino, G. \& Cataldo, A. MicroRNAs and DNA-damaging drugs in breast cancer: strength in numbers. Front. Oncol. 8, 352 (2018).

51. Jackson, A. L. \& Linsley, P. S. Recognizing and avoiding siRNA off-target effects for target identification and therapeutic application. Nat. Rev. Drug Discov. 9 57-67 (2010). 